\title{
Impact of honey on radiotherapy-induced oral mucositis in patients with head and neck cancer: a systematic review and meta-analysis
}

\author{
Xu Tian ${ }^{1}$, Lingli Xu ${ }^{2}$, Xiaoling Liu ${ }^{1}$, Carol Chunfeng Wang ${ }^{3}$, Wei Xie ${ }^{4}$, María F. Jiménez-Herrera ${ }^{5}$, \\ Weiqing Chen ${ }^{1}$
}

${ }^{1}$ Department of Gastroenterology, ${ }^{2}$ Department of Nursing, Chongqing University Cancer Hospital, Chongqing, China; ${ }^{3}$ School of Nursing and Midwifery, Edith Cowan University, Perth, Australia; ${ }^{4}$ Department of Nursing, First Affiliated Hospital of Guizhou University of Traditional Chinese Medicine, Guizhou, China; ${ }^{5}$ Nursing Department, Universitat Rovira i Virgili, Tarragona, Spain

Contributions: (I) Conception and design: X Tian, MF Jiménez-Herrera, W Chen; (II) Administrative support: MF Jiménez-Herrera, W Chen; (III) Provision of study materials or patients: X Tian, L Xu; (IV) Collection and assembly of data: X Tian, X Liu; (V) Data analysis and interpretation: X Tian, W Xie; (VI) Manuscript writing: All authors; (VII) Final approval of manuscript: All authors.

Correspondence to: Dr. María F. Jiménez-Herrera. Nursing Department, Universitat Rovira i Virgili, Avinguda Catalunya, 3543002 Tarragona, Spain. Email: maria.jimenez@urv.cat; Prof. Weiqing Chen. Department of Gastroenterology, Chongqing University Cancer Hospital, Chongqing 400030, China. Email: CQCH_ChenWQ@163.com.

Background: Oral mucositis is one of the most frequent, irreversible and distressing complications faced by head and neck cancer (HNC) patients undergoing radiotherapy. Several studies have investigated the role of honey in the prevention and alleviation of radiation-induced oral mucositis in HNC patients, however, a definitive conclusion has not yet been generated. We performed this updated systematic review and metaanalysis to determine whether honey can prevent and alleviate radiation-induced oral mucositis in HNC patients.

Methods: We searched PubMed, EMBASE, Cochrane Central Register of Controlled Trials (CENTRAL) and China National Knowledge Infrastructure (CNKI) through October 2019. We searched and selected literature, extracted data and assessed risk of bias accordingly, and then conducted statistical analyses with RevMan software version 5.3.

Results: Seven trials involving 412 patients were included in the final analysis. Meta-analyses showed that honey did not decrease the incidence of radiation-induced oral mucositis [(relative risk (RR), 0.69; 95\% confidence interval (CI), 0.40-1.18; $\mathrm{P}=0.18$ ]; however, relieved the severity of oral mucositis $(\mathrm{RR}, 0.22$; 95\% CI, 0.13-0.38; $\mathrm{P}<0.001)$, maintained or increased weight (RR, 1.92; 95\% CI, 1.33-2.77; $\mathrm{P}<0.001)$ and reduced the treatment interruption related to oral mucositis (RR, 0.13; 95\% CI, 0.02-0.97; P=0.05). Qualitative analysis also revealed a decreased incidence of oral mucositis in the honey group.

Conclusions: Based on limited evidence, honey may have a clinical benefit against radiation-induced oral mucositis in HNC patients. However, future trials with large-scale and rigorous methods are warranted to further establish the role of honey in the management of radiation-induced oral mucositis.

Keywords: Head and neck cancer; radiotherapy; oral mucositis; honey; meta-analysis

Submitted Jan 04, 2020. Accepted for publication May 20, 2020.

doi: 10.21037/apm-20-44

View this article at: http://dx.doi.org/10.21037/apm-20-44 


\section{Introduction}

Head and neck cancers (HNC) are the sixth most common cancer around the world (1), with estimated new cases of 710,000 in 2018 (2). Radiotherapy (RT) alone or in combination with chemotherapy or surgery remains a cornerstone in treating HNC (3). Unfortunately, RT also damages epithelium and submucosal connective tissue and then causes inflammation (4). Cancer patients will experience oral mucositis when radiation destructed oral mucosal integrity (5).

Oral mucositis has been emerging as the most frequent, inevitable and distressing complication faced by HNC patients treated with RT (6). Published studies revealed that almost of all HNC patients undergoing RT would experience oral mucositis (7). Evidence also suggested that about half of HNC patients experience grade 3 and above oral mucositis if exposure of the large mucosal surface to 66-70 Gy radiation in 6-7 weeks with 1.8-2 Gy per fraction (8-10). Oral mucositis leads to pain, difficulty swallowing, nutritional issues, increased risk of infection and treatment interruption. It significantly impacts on patients' quality of life (QoL) (11). In addition, severe mucositis prolongs the hospital stay and increases medical expenditure (12). Thus, it is critical to develop effective methods to prevent and treat oral mucositis because the treatment interruption jeopardizes the cancer-destroying effect of RT and magnifies the chance of tumor cell repopulation (13).

Numerous pharmacological and non-pharmacological interventions have been developed to treat radiationinduced oral mucositis (14), such as oral zinc sulfate (15) and laser therapy (16). However, a definitive intervention regime has not yet been established $(17,18)$. Therefore, it is essential to explore alternative agent. Honey is the by-product of flower nectar and the upper aero-digestive tract of the honeybee, which becomes concentrated by the dehydration process inside the beehive (5). As an ancient medicament, honey has been established effective in several fields, such as burn wounds, oral infections and surgical wound $(19,20)$. To date, numerous clinical trials have been designed to explore the role of honey in preventing and treating radiation-induced oral mucositis in HNC patients.

Several systematic reviews and meta-analyses comprehensively evaluated the efficacy of honey in the management of radiation-induced oral mucositis (21-28). However, most of these reviews did not distinguish between RT and chemoradiotherapy. Out of these reviews, only one published in 2013 specifically investigate the role of honey for managing oral mucositis in HNC patients who were treated with RT alone (21). Regrettably the review included two ineligible trials in which patients were treated with combination regime of RT plus chemotherapy. Yarom et al. performed a systematic review to summarize the available evidence of natural agents in the prevention and treatment of oral mucositis in cancer patients, and concluded no guideline was possible (29). So, it remains unclear whether honey use can prevent and treat oral mucositis in HNC patients receiving $\mathrm{RT}$. It is essential to investigate the efficacy of honey in the management of radiation-induced oral mucositis separately because the differences between RT and chemotherapy cannot be ignored (30). We consequently designed this updated systematic review and meta-analysis to accurately determine the role of honey in the prevention and treatment of radiation-induced oral mucositis in $\mathrm{HNC}$ patients.

\section{Methods}

We performed the current systematic review and metaanalysis according to the criteria proposed by the Cochrane Collaboration (CC) (31). We reported all results following the framework issued in the Preferred Reporting Items for Systematic Reviews and Meta-Analysis (PRISMA) statement (32).

\section{Selection criteria}

We designed the selection criteria in line with the previous review (21), which was strongly associated with our topic: (I) adult HNC patients undergoing RT alone; (II) patients in the treatment group assigned to use honey and patients in the control group took control regime or received no treatment; (III) the incidence of severe oral mucositis (grade 3 and 4) was the primary outcome, and the incidence of oral mucositis regardless of grade, incidence of weight maintenance, incidence of treatment interruption and honey-related adverse events were the secondary outcomes; and (IV) clinical trials including clinical controlled trials (CCTs) and randomized controlled trials (RCTs) were considered to meet our criteria. Conference abstracts with sufficient information were also considered to be eligible. Language of trials was limited to English and Chinese.

A trial will be excluded if at least one of following criteria was covered: (I) trial enrolled HNC patients who were also treated with chemotherapy in addition to RT, (II) trial provided insufficient information to enable a judgment, (III) 
trial enrolled patients who had undergoing oral mucositis treatment previously, and (IV) poor methodology (assessed by XT).

\section{Definition of outcomes}

In the current study, the incidence of radiation-induced oral mucositis was defined as the value of a number of oral mucositis cases, irrespective of the grade, divided by the total number of HNC patients completing the whole study. The severity of oral mucositis was evaluated with a scoring system released by the Radiation Therapy Oncology Group (RTOG), World Health Organization (WHO) or the Oral Mucositis Assessment Scale (OMAS) (25). Grade 3 or 4 is considered intolerable or severe according to the established scales (23). The incidence of weight maintenance and the incidence of treatment interruption related to oral mucositis was defined as the value of a number of static or positive weight gain and study discontinuation divided by the total number of cancer patients completing the whole study respectively.

\section{Identification of eligible literature}

Two investigators were assigned to independently search all citations in four databases including PubMed, EMBASE, Cochrane Central Register of Controlled Trials (CENTRAL) and China National Knowledge Infrastructure (CNKI) from its inception through October 2019. We also hand-checked the reference lists of all eligible trials and topic-related reviews so that any potential trial can be covered. Search strategies were constructed by combining Medical Subject Headings (MeSH) and text words according to the unique requirements of individual database (33). The key terms used, including: honey, radiation, and oral mucositis. We documented all search strategies of English databases in the Supplementary File. We used Endnote X7.0 software to manage all citations.

\section{Data extraction}

Two investigators were assigned to independently select the eligible trials according to the selection criteria and extract data from each eligible trial with standard sheet. In this study, we extracted the information including first author, publication year, country of leading author, sample size, sex ratio, age of patients, details of intervention regimes, details of radiation dosages, and outcomes of interest. Any disagreement about inclusion and the extraction of the basic information and data were resolved by consulting a third author.

\section{Assessment of risk of bias}

Two investigators were assigned to independently evaluate the risk of bias from the following seven domains: randomization sequence generation, allocation concealment, blinding of participants and personnel, blinding of outcome assessors, incomplete outcome data, selective reporting, and other biases using the Cochrane risk of bias assessment tool (34). The overall quality of the methodology of an individual study was rated according to the matched level between the actual information and the evaluation criteria (35). Any disagreements between two investigators were addressed through consulting a third investigator.

\section{Statistical analysis}

In this review and meta-analysis, all outcomes were dichotomous data, and thus we used risk ratio (RR) with $95 \%$ confidence interval (CI) to estimate it. We used a random-effects model to calculate all estimates because this model considering within and between studies' heterogeneity simultaneously (36). We firstly adopted Q statistic to describe the heterogeneity qualitatively (37). We also used $\mathrm{I}^{2}$ statistic to quantitatively estimate the proportion of the overall variation (38). For a single outcome, we drew the funnel plot to qualitatively inspect the publication bias if the accumulated number of included studies was more than 10 (39). We completed all statistical analyses with the RevMan 5.3 (Copenhagen, Denmark: The Nordic Cochrane Center, The Cochrane Collaboration, 2013).

\section{Results}

\section{Identification and selection of eligible studies}

We captured 144 items after initially searching for targeted electronic databases. We removed 66 duplicats after running 'Find Duplicates' that is embedded in EndNote software. We classified 13 records into potential inclusion file after carefully reviewing the title and abstract of 78 items. We retrieved full-texts of 13 records in order to check the eligibility of each trial further. After excluding ineligible trials according to the following reasons: conference abstract $(n=1)$, duplicates $(n=1)$, and ineligible treatment 


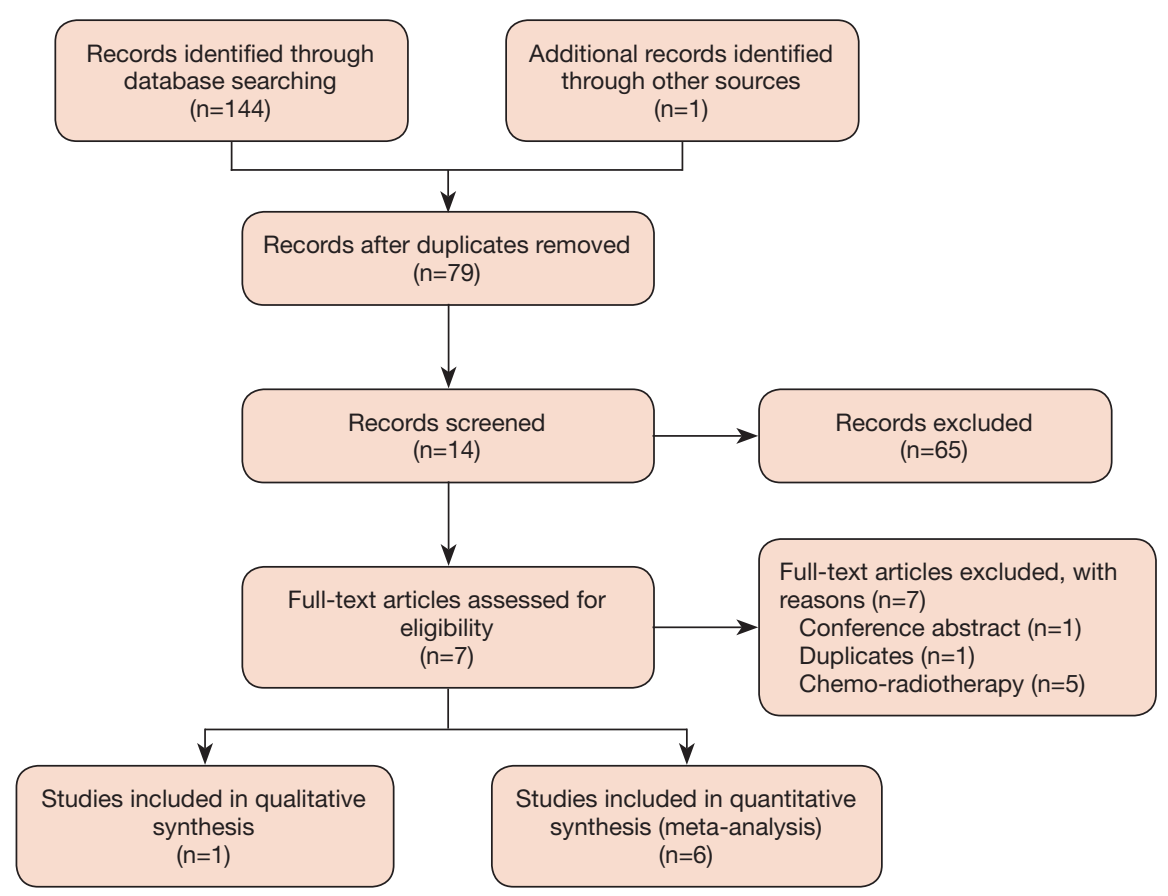

Figure 1 Flow chart of identification and selection of eligible trials. Other sources refers to bibliography lists of eligible studies and topicrelated reviews.

regime ( $\mathrm{n}=5)$, we included $1(40)$ and 6 trials $(5,41-45)$ in the qualitative and quantitative analysis respectively. We drew Figure 1 to depict the process of identification and selection of eligible trials.

\section{Details of characteristics of all eligible trials}

We designed Table 1 to document details of characteristics of all eligible trials. All studies $(5,40-45)$ were published between 2003 and 2017. The sample size of a single trial was ranging from 40 to 82 , with a median sample size of 60 . All trials used honey to prevent and treat radiation-induced oral mucositis. However, control regimes in eligible trials were different. Of 7 trials, 4 used normal saline $(40,41,44,45)$, 1 used lignocaine gel (43), 1 used water mouthwash (42), and 1 used standard protocol (5). Three trials used the Radiation Therapy Oncology Group (RTOG) grading system to evaluate the severity of oral mucositis $(5,40,43)$, 3 used the World Health Organization (WHO) grading system to assess the grade of oral mucositis $(41,42,45)$, and 1 used the Oral Mucositis Assessing Scale (OMAS) to grade the severity of oral mucositis (44). Two trials reported an incidence of radiation-induced oral mucositis $(5,41), 6$ reported incidence of severe radiation-induced oral mucositis (5,41-45), and 2 reported weight maintenance and treatment interruption related to oral mucositis $(5,41)$. All eligible trials reported that baseline information between honey and control groups was not statistically significant.

\section{Assessment of risk of bias of single trial}

We drew Figure 2 to show the details of the risk of bias of an eligible single trial. Of included 7 trials, 5 reported the methods of generating random sequence in detail such as computer-generated random numbers and table of the random numbers $(5,40-43)$. Only 1 trial conducted allocation concealment (43). Two trials stated the information of blinding investigator, participant and assessor $(43,44)$. Furthermore, two trials did not adopt blind method $(5,41)$. In summary, the overall level of quality of all eligible trials was regarded as moderate.

\section{Meta-analysis of clinical outcomes}

\section{Incidence of oral mucositis}

Of seven eligible trials, 2 involving 120 patients reported the incidence of oral mucositis after the patients were instructed to use honey $(5,45)$. Meta-analysis revealed no 


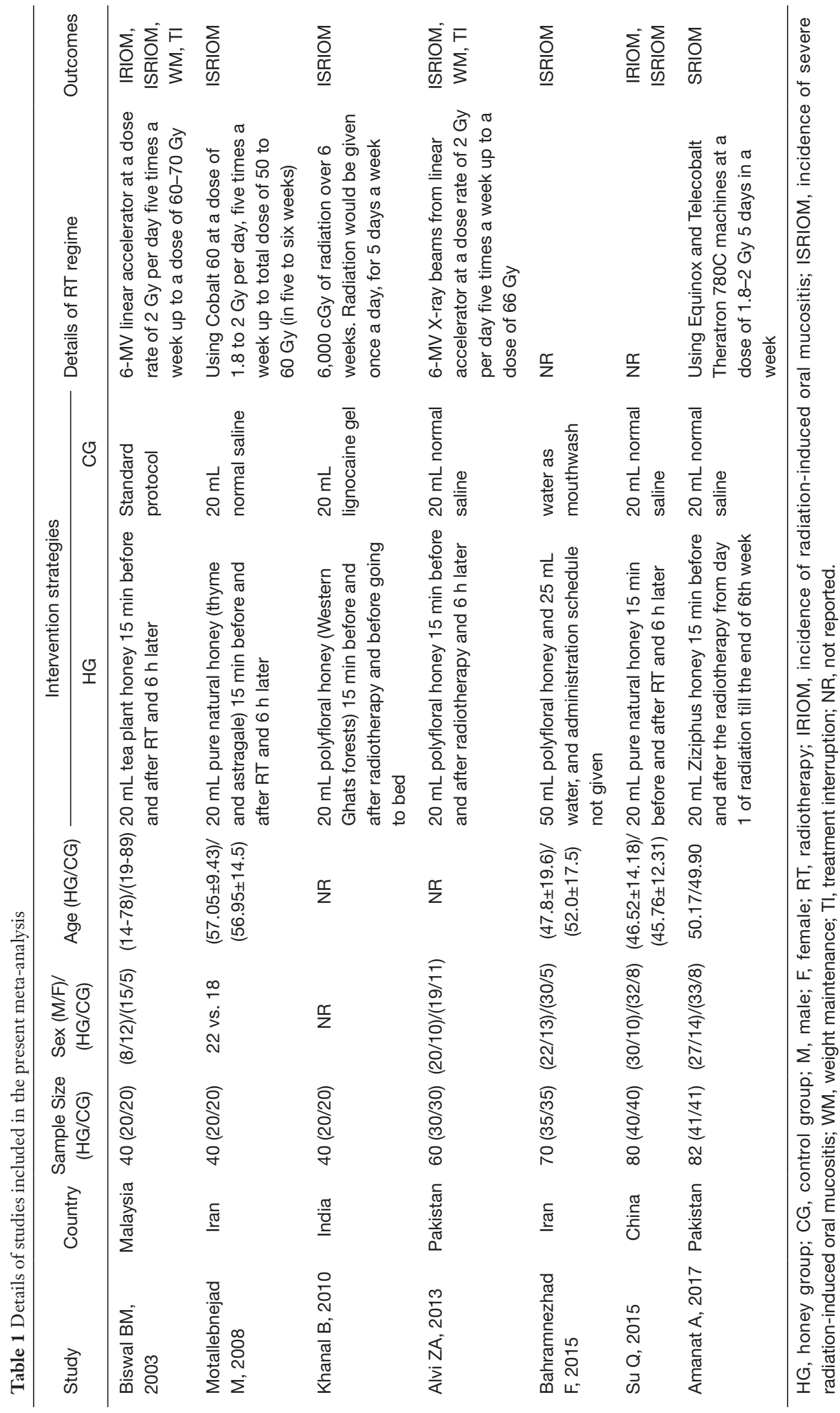




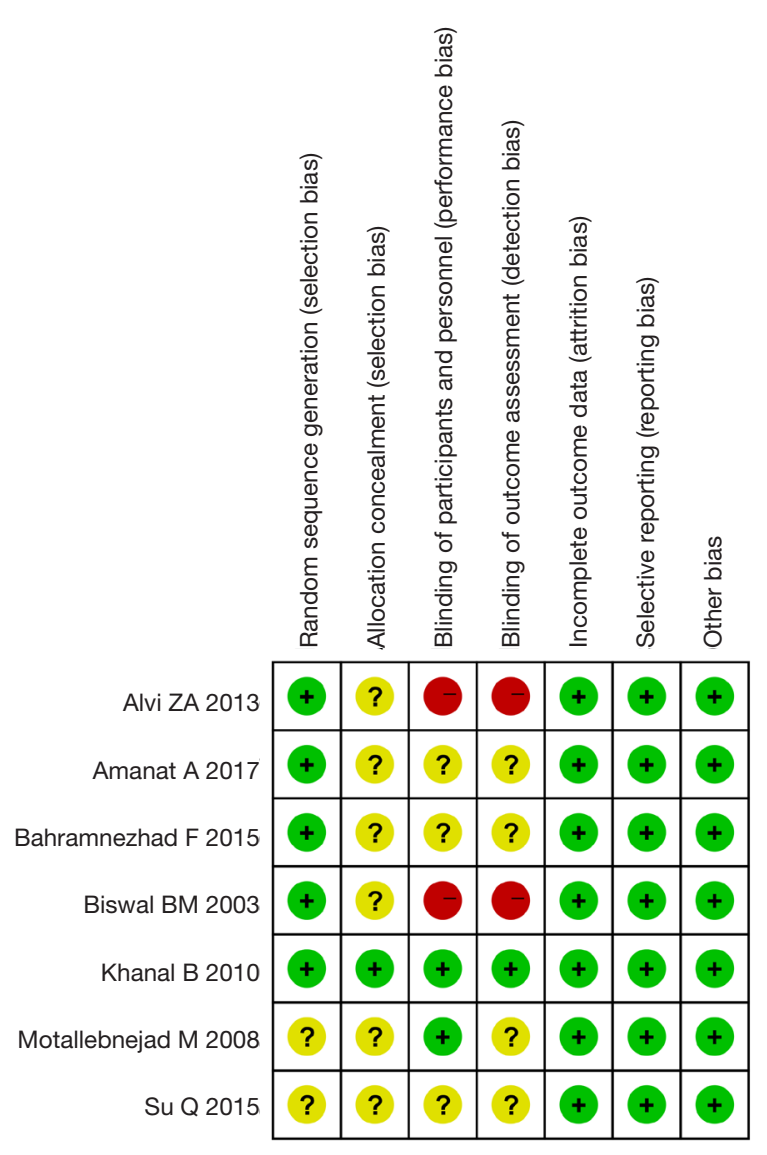

Figure 2 Risk of bias summary. Green, yellow and red solid circle represents low, unclear and high risk of bias respectively.

significant difference in the incidence of oral mucositis between the honey and control groups (two trials; RR, 0.69; $95 \%$ CI, 0.40-1.18; $\mathrm{P}=0.18 ; \mathrm{I}^{2}=81 \%$; Figure 3 A).

\section{Incidence of severe oral mucositis}

Among the seven eligible trials, six studies including 330 patients reported the incidence of severe oral mucositis $(5,41-45)$. Meta-analysis suggested that honey use was associated with decreased incidence of severe oral mucositis (six trials: RR, 0.22; 95\% CI, 0.13-0.38; $\mathrm{P}<0.001, \mathrm{I}^{2}=9 \%$, Figure 3B).

However, one study (40) reported the incidence of severe oral mucositis lesions, which were inconsistent with results in other trials. So, it was not incorporated into metaanalysis. However, we used descriptive analysis to clarify the findings. This trial found that the incidence of severe radiation-induced oral mucositis lesions in the honey group was significantly lower than that in control group $(\mathrm{P}$ value of Grade 3 mucositis: 0.016 and $\mathrm{P}$ value of Grade 4 mucositis: 0.032).

\section{Weight maintenance}

Two trials involving 100 patients, out of seven eligible trials, reported weight maintenance $(5,41)$. Meta-analysis revealed a higher proportion of static or positive weight gain in patients who were instructed to use honey compared to patients were assigned in control group (two trials; RR, 1.92; 95\% CI, 1.33-2.77; $\mathrm{P}<0.001, \mathrm{I}^{2}=0 \%$, Figure 3C).

\section{Oral mucositis induced treatment interruption}

Out of seven eligible trials, two reported the incidence of oral mucositis induced treatment interruption $(5,41)$. The meta-analysis suggested a lower incidence of oral mucositis induced treatment interruption in honey group (two trials; RR, 0.13; 95\% CI, 0.02-0.97; $\mathrm{P}=0.05, \mathrm{I}^{2}=0 \%$, Figure 3D).

\section{Honey related adverse events}

For all eligible trials, no complications related to the administration of honey were reported. Moreover, no report of study discontinuation because of honey.

\section{Publication bias}

In the present systematic review and meta-analysis, the accumulated number of eligible trials for each outcome of interesting were all less than ten. We therefore did not draw a funnel plot to check potential publication bias.

\section{Discussion}

According to the latest data issued by the IARC, HNCs remain the critical threat to publics around the world (2). RT plays an important role in the comprehensive treatment of HNCs (44). Although RT can effectively destroy cancer cells, it also causes damage to healthy tissues located in the radiation portals due to the cytotoxic effect (44). As the most common acute complication of RT, oral mucositis will occur after the mucous membrane integrality was broken (46). Radiation-induced oral mucositis will cause a series of adverse consequences such as pain, discomfort, dysphagia, dehydration, micronutrient deficiency, weight loss (41). To effectively prevent and treat this condition, several intervention regimes have been developed (47). However, an effective, safe and convenient regime is still not established. It is, therefore, necessary to investigate the value of other alternative regimes for the prevention and treatment of radiation-induced oral mucositis in patients 
A

\begin{tabular}{|c|c|c|c|c|c|}
\hline Study or Subgroup & $\begin{array}{l}\text { Honey } \\
\text { Events }\end{array}$ & $\begin{array}{l}y \\
\text { Total }\end{array}$ & $\begin{array}{l}\text { Contr } \\
\text { Events }\end{array}$ & $\begin{array}{l}\text { ol } \\
\text { Total }\end{array}$ & Weight \\
\hline Biswal BM 2003 & 16 & 20 & 19 & 20 & $54.9 \%$ \\
\hline Su Q 2015 & 16 & 40 & 30 & 40 & $45.1 \%$ \\
\hline Total $(95 \% \mathrm{Cl})$ & & 60 & & 60 & $100.0 \%$ \\
\hline Total events & 32 & & 49 & & \\
\hline
\end{tabular}

Risk Ratio

Risk Ratio

H. Random, 95\% Cl Year

M-H, Random, $95 \%$ CI

$0.84[0.66,1.07] 2003$

$0.53[0.35,0.81] 2015$

$0.69[0.40,1.18]$

Test for overall effect: $Z=1.35(P=0.18)$

B

\begin{tabular}{|c|c|c|c|c|c|}
\hline Study or Subgroup & Events & Total & Events & Tota & Weig \\
\hline Biswal BM 2003 & 4 & 20 & 15 & 20 & $29.8 \%$ \\
\hline Motallebnejad M 2008 & 4 & 20 & 16 & 20 & $30.3 \%$ \\
\hline Khanal B 2010 & 1 & 20 & 15 & 20 & $7.6 \%$ \\
\hline Alvi ZA 2013 & 4 & 30 & 12 & 30 & $25.0 \%$ \\
\hline Bahramnezhad F 2015 & 0 & 35 & 6 & 35 & $3.6 \%$ \\
\hline Su Q 2015 & 0 & 40 & 14 & 40 & $3.7 \%$ \\
\hline Total $(95 \% \mathrm{CI})$ & \multicolumn{3}{|c|}{165} & 165 & $100.0 \%$ \\
\hline Total events & \multirow{2}{*}{\multicolumn{5}{|c|}{$\begin{array}{cc}13 & 78 \\
h i^{2}= & 5.47, d f=5(P=0.36) ; l^{2}=9 \%\end{array}$}} \\
\hline Heterogeneity: $\mathrm{Tau}^{2}=$ & & & & & \\
\hline
\end{tabular}

Risk Ratio

M-H, Random, 95\% Cl Year $0.27[0.11,0.66] 2003$ $0.25[0.10,0.62] 2008$ $0.07[0.01,0.46] 2010$ $0.33[0.12,0.92] 2013$ $0.08[0.00,1.32] 2015$ $0.03[0.00,0.56] 2015$

$0.22[0.13,0.38]$

Test for overall effect: $Z=5.45(P<0.00001)$

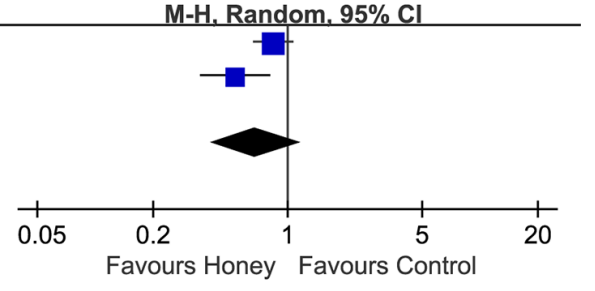

\begin{tabular}{|c|c|c|c|c|c|}
\hline Study or Subgroup & $\begin{array}{l}\text { Honey } \\
\text { Events }\end{array}$ & $\begin{array}{l}y \\
\text { Total } \\
\end{array}$ & $\begin{array}{c}\text { Contr } \\
\text { Events }\end{array}$ & $\begin{array}{l}\text { ol } \\
\text { Total }\end{array}$ & Weight \\
\hline Biswal BM 2003 & 11 & 20 & 5 & 20 & $18.5 \%$ \\
\hline Alvi ZA 2013 & 26 & 30 & 14 & 30 & $81.5 \%$ \\
\hline Total $(95 \% \mathrm{Cl})$ & & 50 & & 50 & $100.0 \%$ \\
\hline Total events & 37 & & 19 & & \\
\hline $\begin{array}{l}\text { Heterogeneity: } \mathrm{Tau}^{2} \\
\text { Test for overall effect }\end{array}$ & $\begin{array}{l}.00 ; \mathrm{Chi}^{2}= \\
=3.46(\mathrm{P}\end{array}$ & $\begin{array}{l}=0.13, \\
=0.00\end{array}$ & $\begin{array}{l}\mathrm{df}=1(\mathrm{P} \\
005)\end{array}$ & $=0.72$ & 2); $\left.\right|^{2}=0 \%$ \\
\hline
\end{tabular}

Risk Ratio

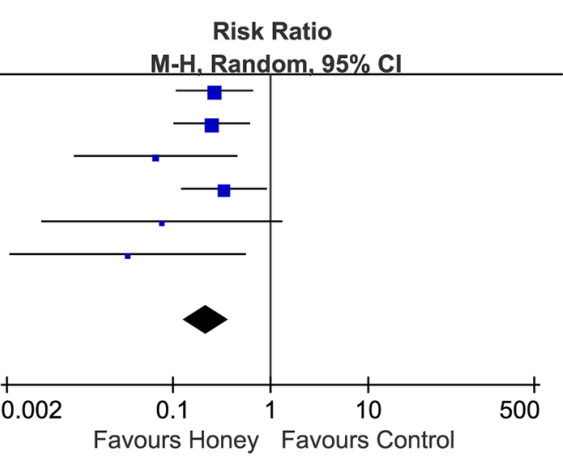

Risk Ratio

M-H. Random. $95 \% \mathrm{Cl}$

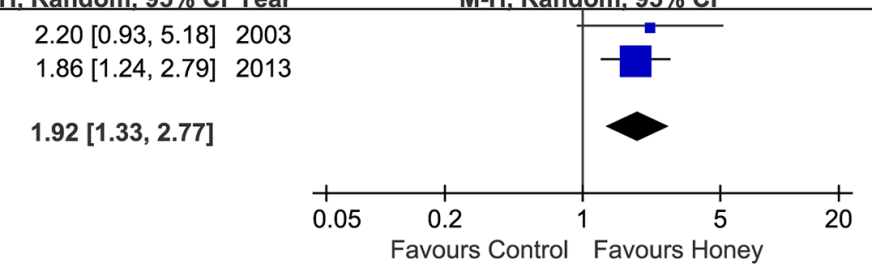

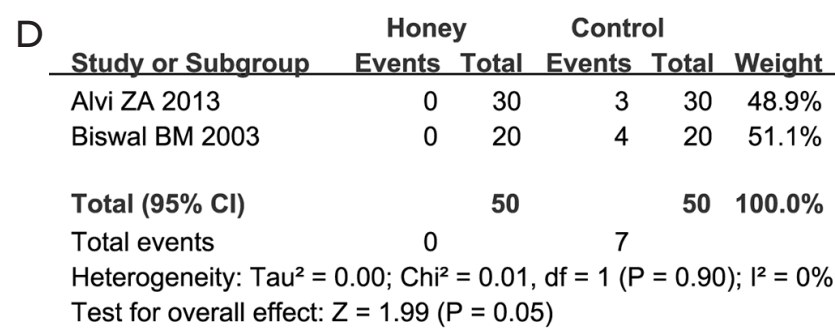

Risk Ratio Risk Ratio

M-H, Random, 95\% Cl

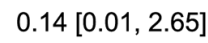

$0.11[0.01,1.94]$

$0.13[0.02,0.97]$

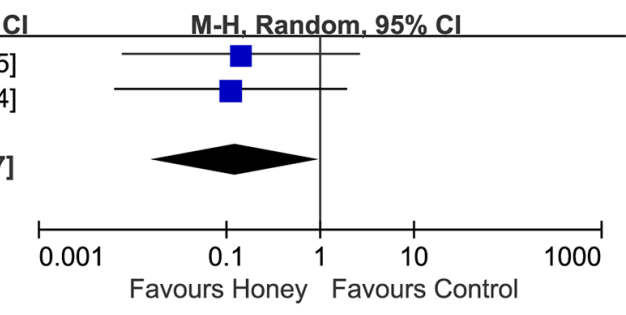

Figure 3 Meta-analysis of clinical outcomes. (A) incidence of oral mucositis, (B) incidence of severe oral mucositis, (C) incidence of weight maintenance, and (D) incidence of treatment interruption. CI, confidence interval; M-H, Mantel Haenszel.

with HNC.

Honey has been used as a prophylactic or therapeutic agent since ancient times (48). Experimental studies suggested that honey has the potential of maintaining integrity of cellular epithelium tissue, preventing the intercellular rupture and inhibiting bacterial growth because of its high viscosity, acidic $\mathrm{PH}$, hydrogen peroxide, high osmolarity, and rich nutritional properties $(43,49)$. According to the findings from several RCTs, honey can enhance healing of minor burns compared with conventional methods such as polyurethane dressings and silver sulfadiazine dressing (48-52). In this review, we first elucidate the efficacy and safety of honey to prevent and treat radiation-induced oral mucositis in HNC patients. Our meta-analysis suggested that honey could significantly decrease the incidence of severe radiation-induced oral mucositis (RR, 0.22; 95\% 
CI, 0.13-0.38; $\mathrm{P}<0.001)$. Moreover, our qualitative analysis also suggested a decreased incidence of severe radiationinduced oral mucositis in the honey group ( $\mathrm{P}$ value of Grade 3 mucositis: 0.016 and $\mathrm{P}$ value of Grade 4 mucositis: 0.032). Additionally, the meta-analysis also found that honey did significantly maintain the weight of patients with $\mathrm{HNC}$ (RR, 1.92; 95\% CI, 1.33-2.77; $\mathrm{P}<0.001)$ and decrease the incidence of treatment interruption related to oral mucositis (RR, 0.13; 95\% CI, 0.02-0.97; $\mathrm{P}=0.05$ ). Several confounding factors such as baseline nutritional status were reported in included studies. So the result of weight maintenance must be cautiously interpreted.

To date, nine systematic reviews and meta-analyses have been conducted to explore the role of honey in preventing and treating radiation- or chemoradiation-induced oral mucositis in cancers. However, a conclusive finding for $\mathrm{HNC}$ patients has not yet been generated. Song and colleagues, in 2012, performed a systematic review and meta-analysis to investigate the protective effects against radiation-induced oral mucositis in HNC, and demonstrated that honey use reduced the incidence of oral mucositis (25). This study enrolled three eligible trials, however one out of three trials recruited most patients receiving chemo-radiotherapy (53). Thus, five potential trials were missed $(40-42,44,45)$. In 2013, a systematic review of five trials, performed by Charalambous et al. investigating the effectiveness of honey for the management of RT-induced oral mucositis in HNC patients, and found a clinical benefit of honey to radiationinduced oral mucositis (21). Unfortunately, two trials involved patients who were prescribed to the combination treatment of RT and chemotherapy instead of RT alone $(53,54)$, and quantitative analysis was also not performed. As a result, four eligible trials were not considered $(40-42,45)$. In 2015, Cho et al. conducted a meta-analysis of nine eligible trials to explore the effects of honey on oral mucositis in HNC patients. However, only three trials of prescribing RT alone to patients were included. It is noted that this review performed subgroup analysis to separately inveterate the impact of honey on patients undergoing RT alone, and revealed that honey had a significantly higher effect in preventing moderate to severe mucositis (28). Unfortunately, this study enrolled an ineligible trial which was published in 2013 (41). In 2016, Co and colleagues performed a meta-analysis of five trials to quantitatively investigate the effect of honey on clinical outcomes among HNC patients receiving RT (22). However, among the five trials, only four prescribed RT. Furthermore, the review failed to capture three potentially eligible trials (22). In the same year of the review published, $\mathrm{Xu}$ et al. designed a meta-analysis to investigate the effects of honey on the management of radio/ chemotherapy-induced mucositis, and revealed that honey could effectively reduce the incidence of oral mucositis (26). Although the authors included seven trials in the final analysis, only three enrolled patients receiving RT alone, and regrettably, the other four trials were misleading $(40-42,45)$. Furthermore, the study enrolled a trial investigating essential oils of manuka (Leptospermum scoparium) and kanuka (Kunzea ericoides) rather than manuka or kanuka honey (55). Yang et al. (2018) performed a network meta-analysis to explore the comparative efficacy of various kinds of honey for chemo/radiotherapy-induced oral mucositis (27). Although this study included twenty-one trials and designed several subgroup analyses according to the age of patients, cancer type and treatment regime, it is still difficult to determine the role of honey use in preventing and treating RT-induced oral mucositis in HNCs because of only eight trials focused on patients receiving RT or chemo/radiotherapy. Liu and colleagues (2019) performed a meta-analysis to investigate the impact of honey use on radiochemotherapy-induced mucositis. In this study, authors considered several cancer types such as HNCs, acute lymphoblastic leukemia (2 trials) and acute myeloid leukemia ( 1 trial). Moreover, it also enrolled 4 trials that participants were pediatric patients with HNC. Another review of 17 trials performed by Münstedt and colleagues evaluated the role of honey in the management of side effects of RT- or radio/chemotherapy-induced oral mucositis (24). In this study, the authors used a qualitative approach to summarize all evidence. Unfortunately, however, trials prescribing RT alone were not analyzed separately. Moreover, the review failed to include one eligible trial published in the Chinese language. In 2013, Yarom et al. on behalf of the Mucositis Study Group of the Multinational Association of Supportive Care in Cancer and International Society of Oral Oncology (MASCC/ISOO) systematically documented the evidence for the use of natural agents for the management of oral mucositis and concluded that the available data were insufficient to reach a consensus (29). Moreover, this review considered an ineligible study that enrolled patients receiving chemo-radiotherapy (53). Compare to these reviews, the present study has two advantages. Firstly, seven eligible trials were analyzed, and this enables robust and reliable findings; secondly, our review only included trials that were specifically focusing on patients receiving RT alone. Thus, the risk of impairing the validity and reliability of pooled results of honey for the efficacy and safety of oral 
mucositis was reduced.

In the present systematic review and meta-analysis, we captured seven eligible trials with an accumulated sample size of 412 patients to generate more robust pooled results. However, some limitations of our study must be acknowledged. Firstly, three instruments were used to grade the severity of radiation-induced oral mucositis across trials; this variation may impair the comparability of these results. A consistent instrument, therefore, should be adopted in future trials. Secondly, control regimes used in the included trials were different including normal saline, lignocaine gel, water and blank control. Inappropriate control regime may increase the risk of performance bias and thus decrease the reliability of findings. Therefore, we suggest using a uniform control regime in future trials such as normal saline. Thirdly, all analyzed trials focused on the clinical outcomes such as incidence of intolerable radiation-induced oral mucositis, however other outcomes from patients' perspective such as quality of life were not investigated. Therefore, more outcomes should be designed in future trials. Fourthly, the sample size of individual eligible trial was insufficient, and thus future trials with large scale and rigorous methodology must be considered. Fifthly, our review did not address confounding variables for analyzing weight maintenance and treatment interruption related to oral mucositis due to insufficient data. So the pooled results for these outcomes should be cautiously interpreted. More importantly, future trials should report detailedly baseline variables. Sixthly, our review did not design subgroup analysis according to the RT machines because sufficient information cannot be extracted. We therefore suggest future trials to describe the details of RT.

In conclusion, oral honey may have the potential of relieving the severity of radiation-induced oral mucositis, maintaining weight, and decreasing the incidence of treatment interruption related to oral mucositis in patients with HNC based on the limited evidence. Considering limitations in all included trials, we suggest designing future trials with large-scales and rigorous methods to further establish the role of oral honey for radiation-induced oral mucositis.

\section{Acknowledgments}

We would like to express our sincere appreciation to authors who performed all eligible studies included in the present study.

Funding: None.

\section{Footnote}

Conflicts of Interest: All authors have completed the ICMJE uniform disclosure form (available at http://dx.doi. org/10.21037/apm-20-404). The authors have no conflicts of interest to declare.

Etbical Statement: The authors are accountable for all aspects of the work in ensuring that questions related to the accuracy or integrity of any part of the work are appropriately investigated and resolved.

Open Access Statement: This is an Open Access article distributed in accordance with the Creative Commons Attribution-NonCommercial-NoDerivs 4.0 International License (CC BY-NC-ND 4.0), which permits the noncommercial replication and distribution of the article with the strict proviso that no changes or edits are made and the original work is properly cited (including links to both the formal publication through the relevant DOI and the license). See: https://creativecommons.org/licenses/by-nc-nd/4.0/.

\section{References}

1. Taghipour M, Sheikhbahaei S, Marashdeh W, et al. Use of 18F-Fludeoxyglucose-Positron Emission Tomography/ Computed Tomography for Patient Management and Outcome in Oropharyngeal Squamous Cell Carcinoma: A Review. JAMA Otolaryngol Head Neck Surg 2016;142:79-85.

2. Bray F, Ferlay J, Soerjomataram I, et al. Global cancer statistics 2018: GLOBOCAN estimates of incidence and mortality worldwide for 36 cancers in 185 countries. CA Cancer J Clin 2018;68:394-424.

3. Paiar F, Cristaudo A, Gonnelli A, et al. Radiation-induced nausea and vomiting in head and neck cancer: Is it something worth considering in the intensity modulated radiotherapy era? "A narrative review". Head Neck 2020;42:131-7.

4. Baker DG. The radiobiological basis for tissue reactions in the oral cavity following therapeutic $x$-irradiation. A review. Arch Otolaryngol 1982;108:21-4.

5. Biswal BM, Zakaria A, Ahmad NM. Topical application of honey in the management of radiation mucositis: a preliminary study. Support Care Cancer 2003;11:242-8.

6. Rodriguez-Caballero A, Torres-Lagares D, Robles-Garcia $\mathrm{M}$, et al. Cancer treatment-induced oral mucositis: a critical review. Int J Oral Maxillofac Surg 2012;41:225-38. 
7. Vera-Llonch M, Oster G, Hagiwara M, et al. Oral mucositis in patients undergoing radiation treatment for head and neck carcinoma. Cancer 2006;106:329-36.

8. Horiot JC, Bontemps $\mathrm{P}$, van den Bogaert $\mathrm{W}$, et al. Accelerated fractionation (AF) compared to conventional fractionation (CF) improves loco-regional control in the radiotherapy of advanced head and neck cancers: results of the EORTC 22851 randomized trial. Radiother Oncol 1997;44:111-21.

9. Horiot JC, Le Fur R, N'Guyen T, et al. Hyperfractionation versus conventional fractionation in oropharyngeal carcinoma: final analysis of a randomized trial of the EORTC cooperative group of radiotherapy. Radiother Oncol 1992;25:231-41.

10. Lee DJ, Cosmatos D, Marcial VA, et al. Results of an RTOG phase III trial (RTOG 85-27) comparing radiotherapy plus etanidazole with radiotherapy alone for locally advanced head and neck carcinomas. Int J Radiat Oncol Biol Phys 1995;32:567-76.

11. Mercadante S, Aielli F, Adile C, et al. Prevalence of oral mucositis, dry mouth, and dysphagia in advanced cancer patients. Support Care Cancer 2015;23:3249-55.

12. Murphy BA, Ridner S, Wells N, et al. Quality of life research in head and neck cancer: a review of the current state of the science. Crit Rev Oncol Hematol 2007;62:251-67.

13. Shih A, Miaskowski C, Dodd MJ, et al. Mechanisms for radiation-induced oral mucositis and the consequences. Cancer Nurs 2003;26:222-9.

14. Kobya Bulut H, Guducu Tufekci F. Honey prevents oral mocositis in children undergoing chemotherapy: A quasiexperimental study with a control group. Complement Ther Med 2016;29:132-40.

15. Tian X, Liu XL, Pi YP, et al. Oral Zinc Sulfate for Prevention and Treatment of Chemotherapy-Induced Oral Mucositis: A Meta-Analysis of Five Randomized Controlled Trials. Front Oncol 2018;8:484.

16. Lalla RV, Bowen J, Barasch A, et al. MASCC/ISOO clinical practice guidelines for the management of mucositis secondary to cancer therapy. Cancer 2014;120:1453-61.

17. Rambod M, Pasyar N, Ramzi M. The effect of zinc sulfate on prevention, incidence, and severity of mucositis in leukemia patients undergoing chemotherapy. Eur J Oncol Nurs 2018;33:14-21.

18. Villa A, Sonis ST. Mucositis: pathobiology and management. Curr Opin Oncol 2015;27:159-64.

19. Efem SE, Udoh KT, Iwara CI. The antimicrobial spectrum of honey and its clinical significance. Infection
1992;20:227-9.

20. Vardi A, Barzilay Z, Linder N, et al. Local application of honey for treatment of neonatal postoperative wound infection. Acta Paediatr 1998;87:429-32.

21. Charalambous M, Raftopoulos V, Lambrinou E, et al. The effectiveness of honey for the management of radiotherapy-induced oral mucositis in head and neck cancer patients: A systematic review of clinical trials. Eur J Integr Med 2013;5:217-25.

22. Co JL, Mejia MB, Que JC, et al. Effectiveness of honey on radiation-induced oral mucositis, time to mucositis, weight loss, and treatment interruptions among patients with head and neck malignancies: A meta-analysis and systematic review of literature. Head Neck 2016;38:1119-28.

23. Liu TM, Luo YW, Tam KW, et al. Prophylactic and therapeutic effects of honey on radiochemotherapyinduced mucositis: a meta-analysis of randomized controlled trials. Support Care Cancer 2019;27:2361-70.

24. Munstedt K, Momm F, Hubner J. Honey in the management of side effects of radiotherapy- or radio/ chemotherapy-induced oral mucositis. A systematic review. Complement Ther Clin Pract 2019;34:145-52.

25. Song JJ, Twumasi-Ankrah P, Salcido R. Systematic review and meta-analysis on the use of honey to protect from the effects of radiation-induced oral mucositis. Adv Skin Wound Care 2012;25:23-8.

26. Xu JL, Xia R, Sun ZH, et al. Effects of honey use on the management of radio/chemotherapy-induced mucositis: a meta-analysis of randomized controlled trials. Int J Oral Maxillofac Surg 2016;45:1618-25.

27. Yang C, Gong G, Jin E, et al. Topical application of honey in the management of chemo/radiotherapy-induced oral mucositis: A systematic review and network meta-analysis. Int J Nurs Stud 2019;89:80-7.

28. Cho HK, Jeong YM, Lee HS, et al. Effects of honey on oral mucositis in patients with head and neck cancer: A meta-analysis. Laryngoscope 2015;125:2085-92.

29. Yarom N, Ariyawardana A, Hovan A, et al. Systematic review of natural agents for the management of oral mucositis in cancer patients. Support Care Cancer 2013;21:3209-21.

30. Thomsen M, Vitetta L. Adjunctive Treatments for the Prevention of Chemotherapy- and Radiotherapy-Induced Mucositis. Integr Cancer Ther 2018;17:1027-47.

31. Higgins JPT, Green S. Cochrane Handbook for Systematic Reviews of Interventions Version 5.1.0 [updated March 2011]. The Cochrane Collaboration, 2011 Available online: www.handbook.cochrane.org. 
32. Moher D, Liberati A, Tetzlaff J, et al. Preferred reporting items for systematic reviews and meta-analyses: the PRISMA statement. J Clin Epidemiol 2009;62:1006-12.

33. Zhang B, Cui XS. Effect of continuing nursing on self-care ability of diabetic patients: A meta-analysis. TMR Integr Nurs 2019;3:49-55.

34. Higgins JP, Altman DG, Gotzsche PC, et al. The Cochrane Collaboration's tool for assessing risk of bias in randomised trials. Bmj 2011;343:d5928.

35. Huang WQ, Yang Z, Tang DX, et al. Pharmacological intervention of traditional Chinese medicine for the quality of life in patients with colorectal cancer: a systematic review and meta-analysis. Tradit Med Res 2018;3:95-105.

36. DerSimonian R, Laird N. Meta-analysis in clinical trials. Control Clin Trials 1986;7:177-88.

37. Bowden J, Tierney JF, Copas AJ, et al. Quantifying, displaying and accounting for heterogeneity in the metaanalysis of RCTs using standard and generalised Q statistics. BMC Med Res Methodol 2011;11:41.

38. Higgins JP, Thompson SG. Quantifying heterogeneity in a meta-analysis. Stat Med 2002;21:1539-58.

39. Palma Perez S, Delgado Rodriguez M. Practical considerations on detection of publication bias. Gac Sanit 2006;20 Suppl 3:10-6.

40. Amanat A, Ahmed A, Kazmi A, et al. The Effect of Honey on Radiation-induced Oral Mucositis in Head and Neck Cancer Patients. Indian J Palliat Care 2017;23:317-20.

41. Alvi ZA, Mahmood A, Rasul S, et al. Role of honey in prevention of radiation induced mucositis in head and neck cancer. Pak Armed Forces Med J 2013;63:379-83.

42. Bahramnezhad F, Dehghan Nayeri N, Bassampour SS, et al. Honey and Radiation-Induced Stomatitis in Patients With Head and Neck Cancer. Iran Red Crescent Med J 2015;17:e19256.

43. Khanal B, Baliga M, Uppal N. Effect of topical honey on limitation of radiation-induced oral mucositis: an intervention study. Int J Oral Maxillofac Surg 2010;39:1181-5.

44. Motallebnejad M, Akram S, Moghadamnia A, et al. The effect of topical application of pure honey on radiationinduced mucositis: a randomized clinical trial. J Contemp
Dent Pract 2008;9:40-7.

45. Su Q, Li YM, Han PB, et al. Honey as topical prophylaxis against radiochemotherapy-induced oral mucositis in nasopharyngeal carcinoma. Contemp Med 2018;24:55-7.

46. Vissink A, Jansma J, Spijkervet FK, et al. Oral sequelae of head and neck radiotherapy. Crit Rev Oral Biol Med 2003;14:199-212.

47. Shuai T, Tian X, Shi B, et al. Prophylaxis With Oral Zinc Sulfate Against Radiation Induced Oral Mucositis in Patients With Head and Neck Cancers: A Systematic Review and Meta-Analysis of Four Randomized Controlled Trials. Front Oncol 2019;9:165.

48. Subrahmanyam M. Topical application of honey in treatment of burns. Br J Surg 1991;78:497-8.

49. Subrahmanyam $M$. A prospective randomised clinical and histological study of superficial burn wound healing with honey and silver sulfadiazine. Burns 1998;24:157-61.

50. Segovia D. The Clinical Benefits of Active Leptospermum Honey in Oncologic Wounds. Ostomy Wound Manage 2010;56:10-2.

51. Asher A, Mashhood, Tahir A, et al. Honey compared with $1 \%$ silver sulfadiazine cream in the treatment of superficial and partial thickness burns. J Pak Assoc Dermatol 2006;16:14-9.

52. Subrahmanyam $M$, . Honey impregnated gauze versus polyurethane film (OpSite) in the treatment of burns-a prospective randomised study. Br J Plast Surg 1993;46:322-3.

53. Rashad UM, Al-Gezawy SM, El-Gezawy E, et al. Honey as topical prophylaxis against radiochemotherapy-induced mucositis in head and neck cancer. J Laryngol Otol 2009;123:223-8.

54. Bardy J, Molassiotis A, Ryder WD, et al. A double-blind, placebo-controlled, randomised trial of active manuka honey and standard oral care for radiation-induced oral mucositis. Br J Oral Maxillofac Surg 2012;50:221-6.

55. Maddocks-Jennings W, Wilkinson JM, Cavanagh HM, et al. Evaluating the effects of the essential oils Leptospermum scoparium (manuka) and Kunzea ericoides (kanuka) on radiotherapy induced mucositis: a randomized, placebo controlled feasibility study. Eur J Oncol Nurs 2009;13:87-93.
Cite this article as: Tian $\mathrm{X}, \mathrm{Xu} \mathrm{L}$, Liu $\mathrm{X}$, Wang CC, Xie W, Jiménez-Herrera MF, Chen W. Impact of honey on radiotherapy-induced oral mucositis in patients with head and neck cancer: a systematic review and meta-analysis. Ann Palliat Med 2020;9(4):1431-1441. doi: 10.21037/apm-20-44 
Supplementary

\section{Search strategies for English-language databases}

\section{Search strategy of PubMed}

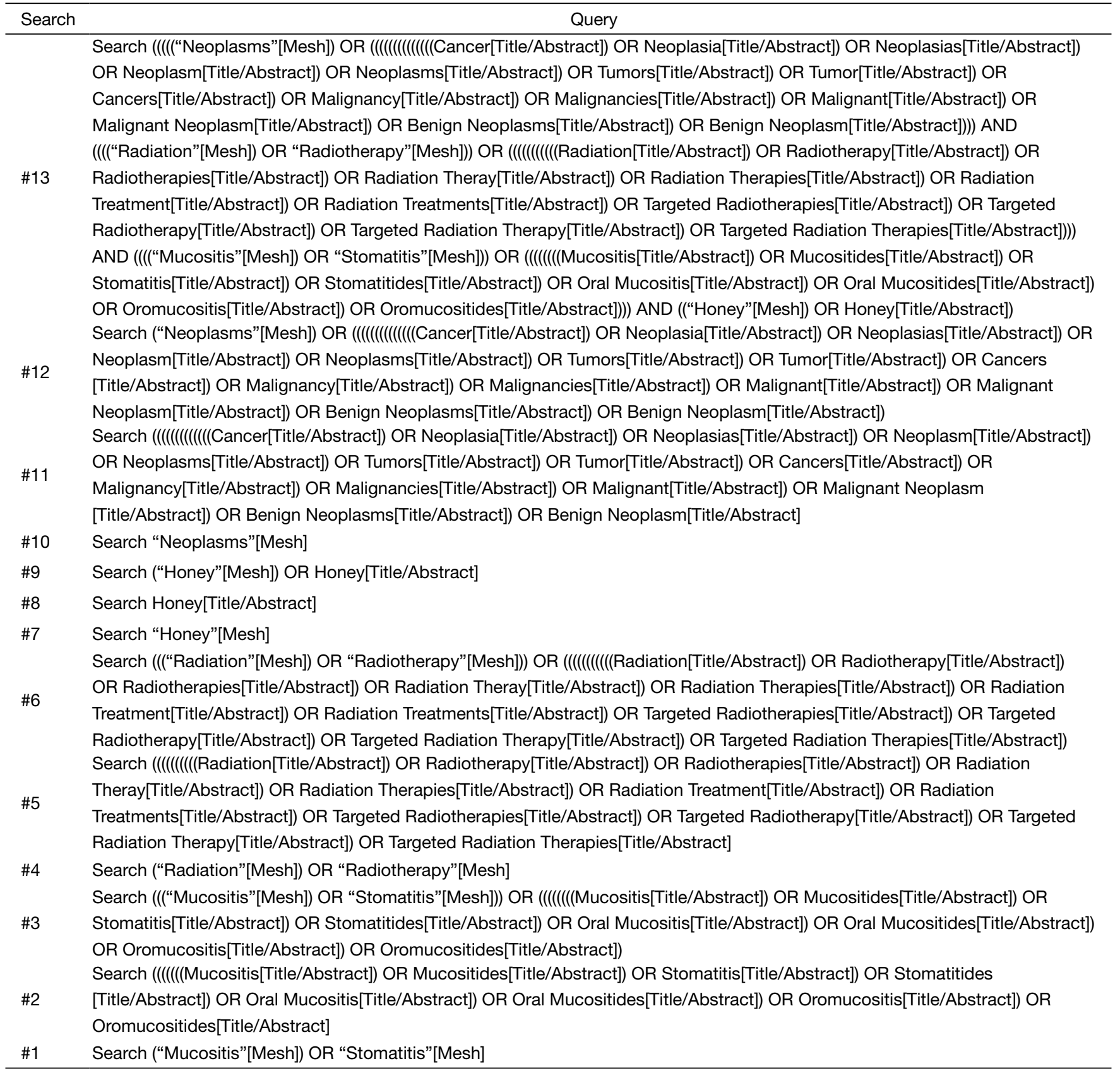

\section{Search strategy of CENTRAL}

Search Name: Honey for Oral mucositis

Last Saved: 19/10/2019 01:07:41

Comment: 
ID Search

\#1 ("mucositis"):ti,ab,kw OR ("mucositides"):ti,ab,kw OR ("stomatitis"):ti,ab,kw OR ("stomatitides"):ti,ab,kw OR ("oral mucositis"):ti,ab,kw (Word variations have been searched)

\#2 MeSH descriptor: [Stomatitis] explode all trees

\#3 MeSH descriptor: [Mucositis] explode all trees

\#4 \#1 OR \#2 OR \#3

\#5 ("radiation"):ti,ab,kw OR ("radiotherapy"):ti,ab,kw OR ("radiotherapies"):ti,ab,kw (Word variations have been searched)

\#6 MeSH descriptor: [Radiation] explode all trees

\#7 MeSH descriptor: [Radiotherapy] explode all trees

\#8 \#5 OR \#6 OR \#7

\#9 ("cancer"):ti,ab,kw OR ("neoplasia"):ti,ab,kw OR ("neoplasm"):ti,ab,kw OR ("tumor"):ti,ab,kw OR ("malignancy"):ti,ab,kw (Word variations have been searched)

\#10 ("malignant"):ti,ab,kw OR ("benign neoplasm"):ti,ab,kw OR ("malignant neoplasm"):ti,ab,kw (Word variations have been searched)

\#11 MeSH descriptor: [Neoplasms] explode all trees

\#12 \#9 OR \#10 OR \#11

\#13 ("honey"):ti,ab,kw (Word variations have been searched)

\#14 MeSH descriptor: [Honey] explode all trees

\#15 \#13 OR \#14

\#16 \#4 AND \#8 AND \#12 AND \#15

\section{Search strategy of Embase}

Embase

Session Results

No. Query Results

\#13. \#3 AND \#6 AND \#9 AND \#12

\#12. \#10 OR \#11

\#11. 'honey'/exp

\#10. honey:ti,ab,kw

\#9. \#7 OR \#8

\#8. 'mucosa inflammation'/exp OR 'oral mucositis'/exp OR 'stomatitis'/exp

\#7. mucositis:ti,ab,kw OR mucositides:ti,ab,kw OR stomatitis:ti,ab,kw OR stomatitides:ti,ab,kw OR 'oral mucositis':ti,ab,kw OR 'oral mucositides':ti,ab,kw OR oromucositis:ti,ab,kw OR oromucositides:ti,ab,kw

\#6. \#4 OR \#5

\#5. 'radiation'/exp OR 'radiotherapy'/exp

\#4. radiation:ti,ab,kw OR radiotherap*:ti,ab,kw OR 'radiation therapy':ti,ab,kw OR 'radiation therapies':ti,ab,kw OR 'radiation treatment':ti,ab,kw OR 'radiation treatments':ti,ab,kw OR 'targeted radiotherapies':ti,ab,kw OR 'targeted radiotherapy':ti,ab,kw OR 'targeted radiation therapy':ti,ab,kw OR 'targeted radiation therapies':ti,ab,kw

\#3. \#1 OR \#2

\#2. 'malignant neoplasm'/exp OR 'neoplasm'/exp

\#1. cancer*:ti,ab,kw OR neoplasia*:ti,ab,kw OR neoplasm*:ti,ab,kw OR tumor*:ti,ab,kw OR malignanc*:ti,ab,kw OR malignant*:ti,ab,kw OR 'malignant neoplasm':ti,ab,kw OR 'malignant neoplasm*':ti,ab,kw OR 'benign neoplasms':ti,ab,kw OR 'benign neoplasm':ti,ab,kw 